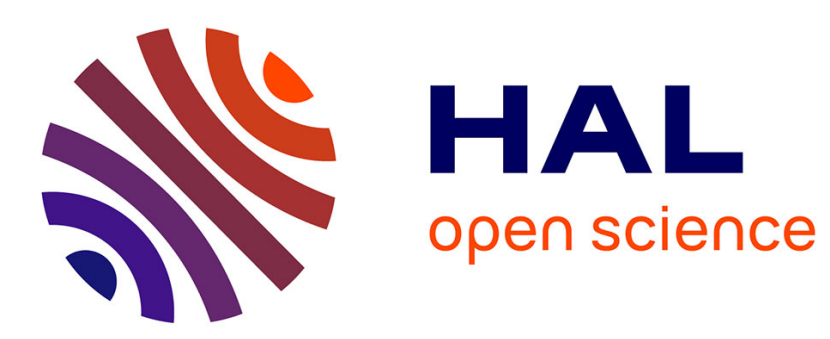

\title{
Efficient decoding of random errors for quantum expander codes
}

\author{
Antoine Grospellier, Anthony Leverrier, Omar Fawzi
}

\section{To cite this version:}

Antoine Grospellier, Anthony Leverrier, Omar Fawzi. Efficient decoding of random errors for quantum expander codes. Journées Informatique Quantique 2017, Nov 2017, Bordeaux, France. pp.521-534. hal-01671491

\section{HAL Id: hal-01671491 \\ https://hal.science/hal-01671491}

Submitted on 22 Dec 2017

HAL is a multi-disciplinary open access archive for the deposit and dissemination of scientific research documents, whether they are published or not. The documents may come from teaching and research institutions in France or abroad, or from public or private research centers.
L'archive ouverte pluridisciplinaire HAL, est destinée au dépôt et à la diffusion de documents scientifiques de niveau recherche, publiés ou non, émanant des établissements d'enseignement et de recherche français ou étrangers, des laboratoires publics ou privés. 


\section{Efficient decoding of random errors for quantum expander codes}

\section{Antoine Grospellier \& Anthony Leverrier \& Omar Fawzi}

10 Novembre 2017

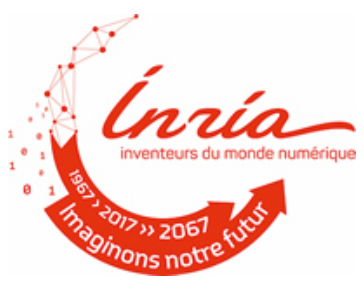



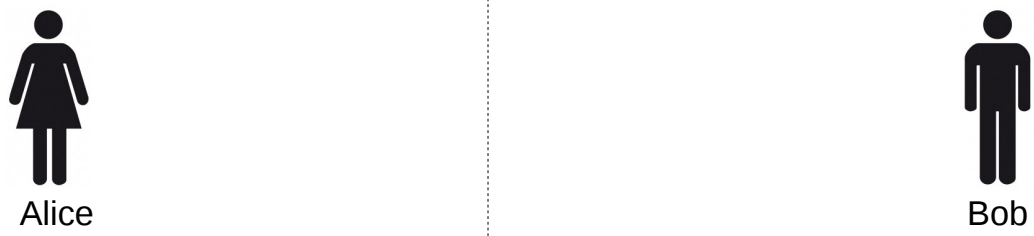
k Q-bits

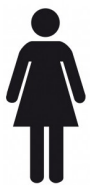

Alice

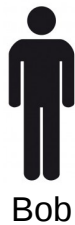



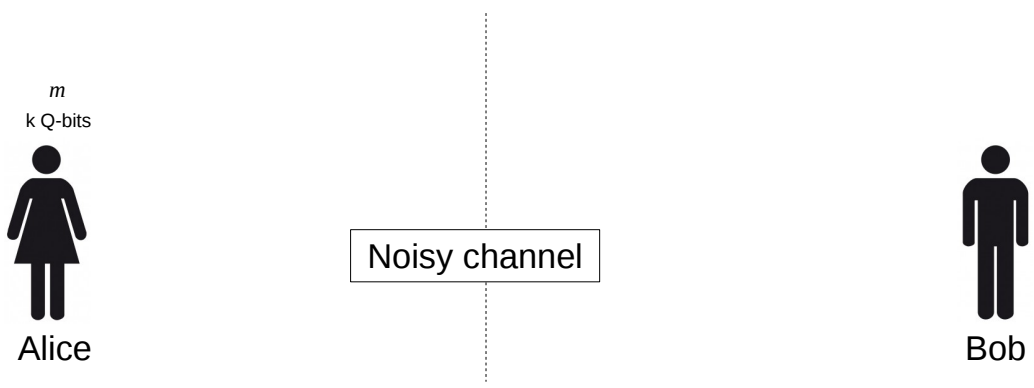


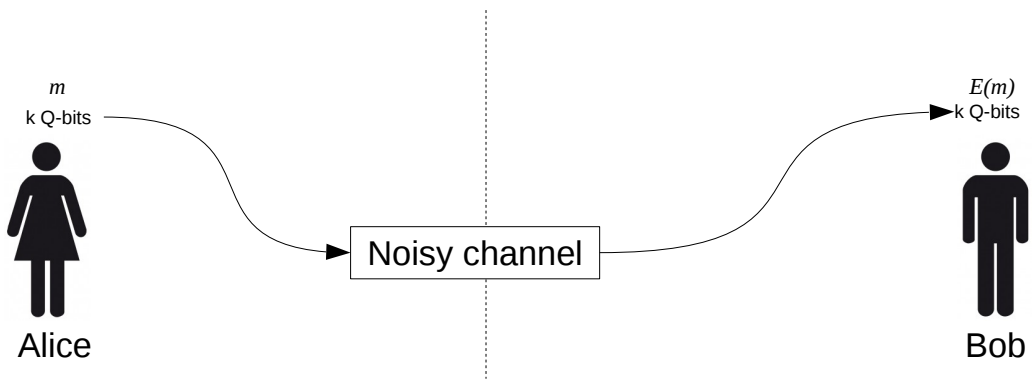




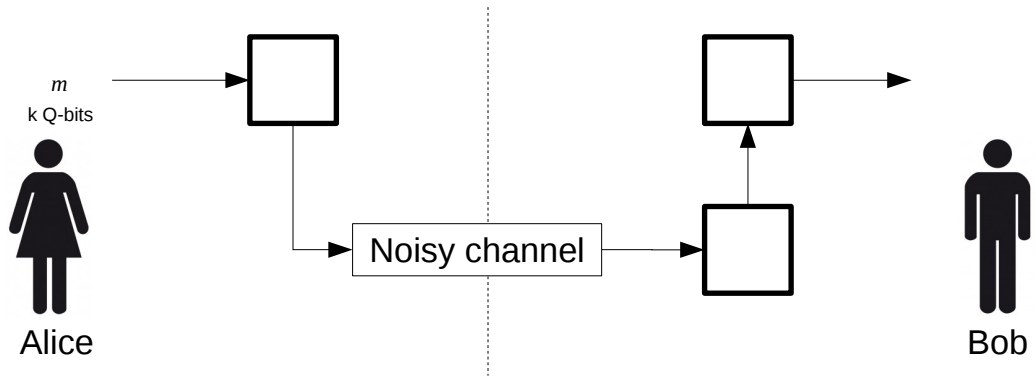




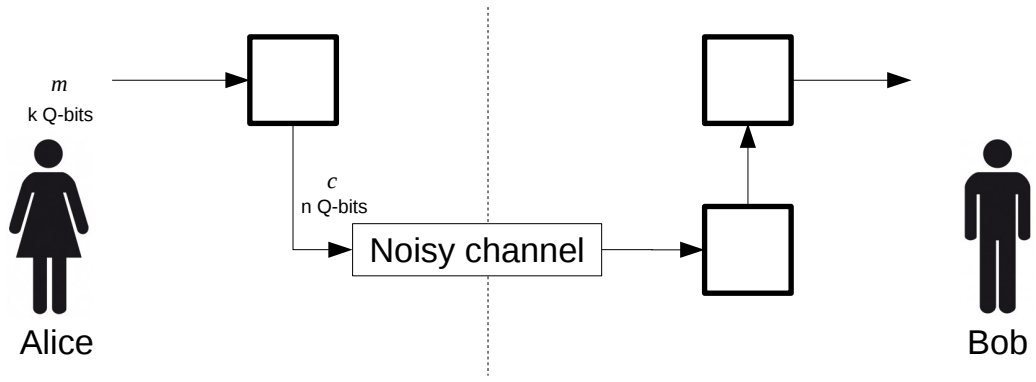




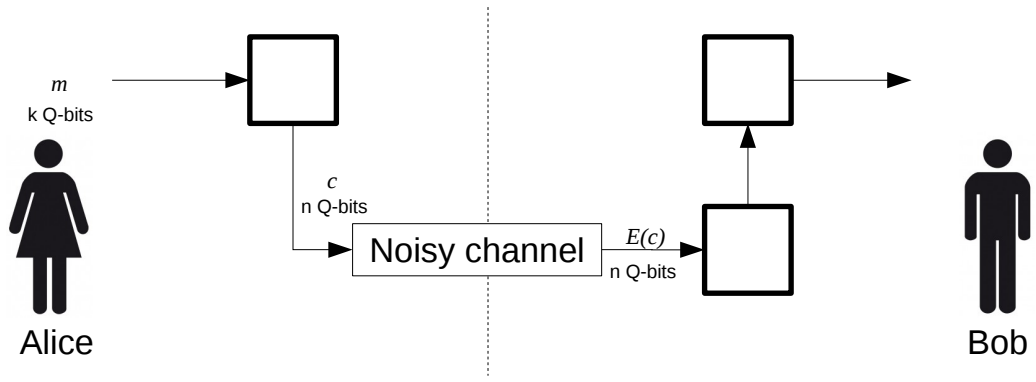



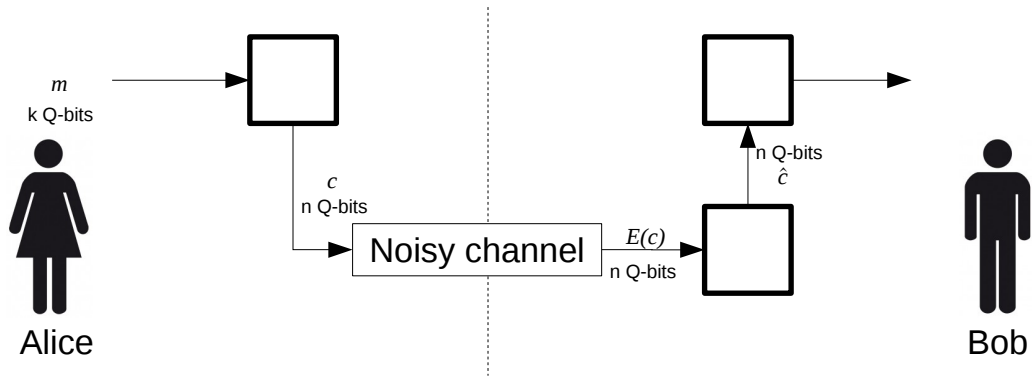


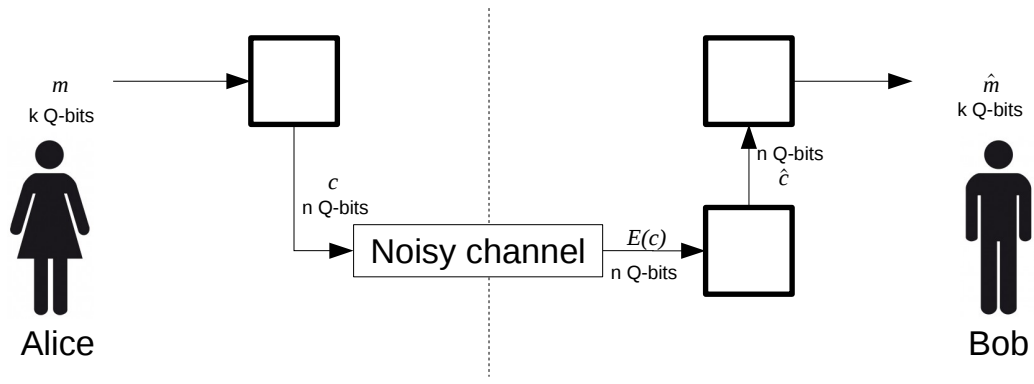




\section{Content of the talk}

The hypergraph product of an expander code :

- is an LDPC quantum code

- has a constant rate

- has a minimal distance : $d=\Theta(\sqrt{n})$

The decoding algorithm :

- has a capacity of correction : $\Theta(\sqrt{n})$

- corrects the error with high probability for the depolarizing channel 


\section{Content of the talk}

(1) Classical expander codes

(2) Quantum expander codes

(3) Our contribution 


\section{Plan}

(1) Classical expander codes

(2) Quantum expander codes

(3) Our contribution 


\section{Tanner graph of a code}

Bits Parity-check nodes

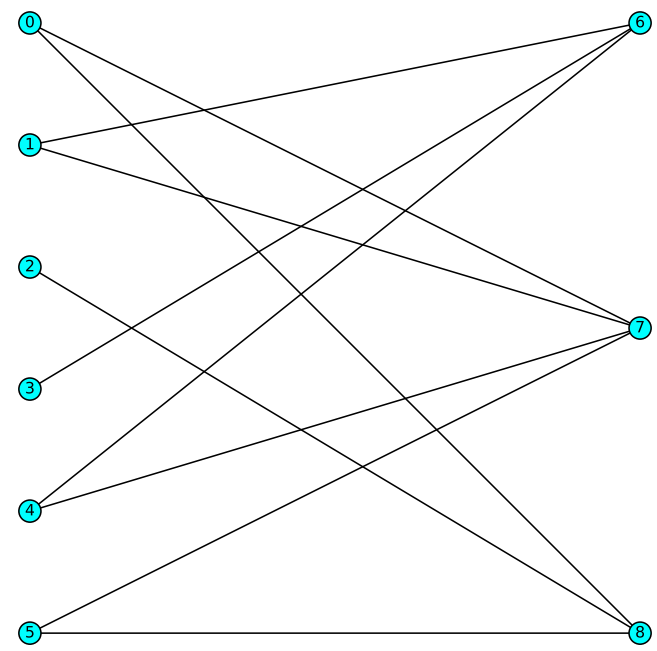




\section{Classical expander codes [Sipser \& Spielman, '96]}

Theorem [Sipser \& Spielman, '96]

We can construct a good family $\left(\mathcal{C}_{n}\right)_{n \in \mathbb{N}}$ of $[n, k, d]$-error correcting codes. Here "Good" means :

- This family is LDPC

- $k$ and $d$ are linear in $n$

- There exists an efficient correcting algorithm

\section{Remark}

Good expander codes can be found efficiently by picking a random biregular graph 


\section{Decoding algorithm}

Bits

Parity-check nodes

- Error:

$e_{0}=\{0,1,2\}$

- Unsatisfied check-nodes (syndrome) : $\{10,12,14,19\}$

- Satisfied check-nodes : $\{11,13,15$, $16,17,18\}$

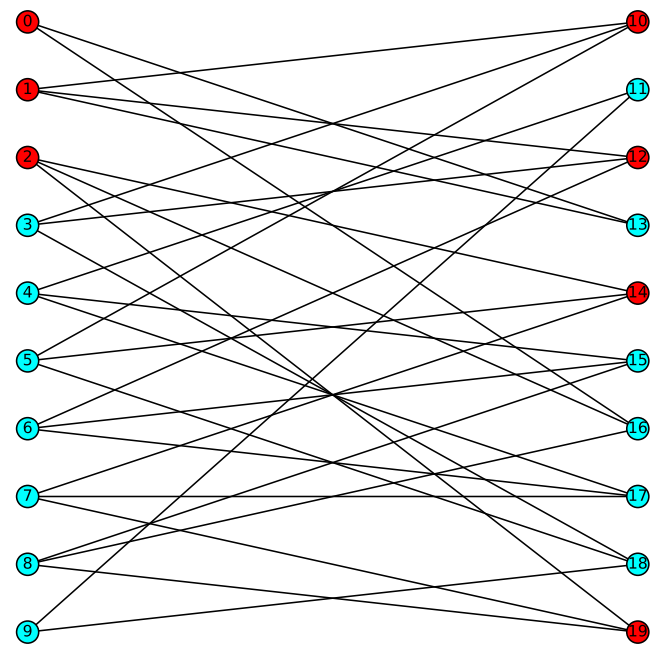




\section{Decoding algorithm}

Bits

Parity-check nodes

- INPUT : syndrome

- The error $e_{0}$ is unknown

- OUTPUT : e a set of bits

- GOAL : $e=e_{0}$

- The algorithm flips a bit when it decreases the syndrome

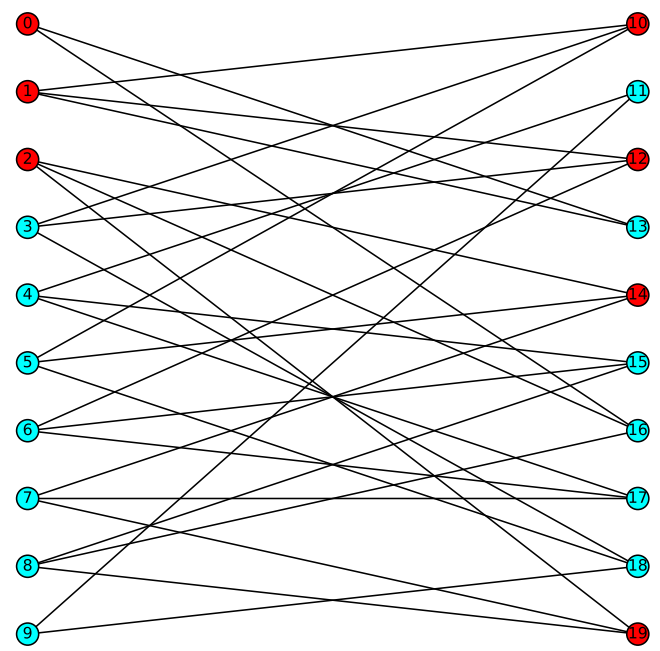




\section{Decoding algorithm : first example}

Bits

Parity-check nodes

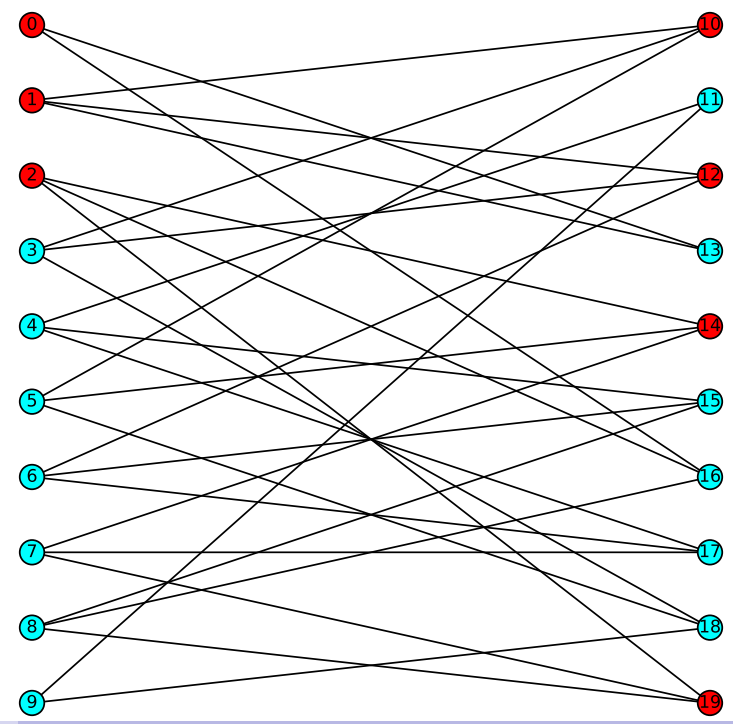

A. Grospellier

Efficient decoding of random errors for quantum expander codes 


\section{Decoding algorithm : first example}

Bits

Parity-check nodes

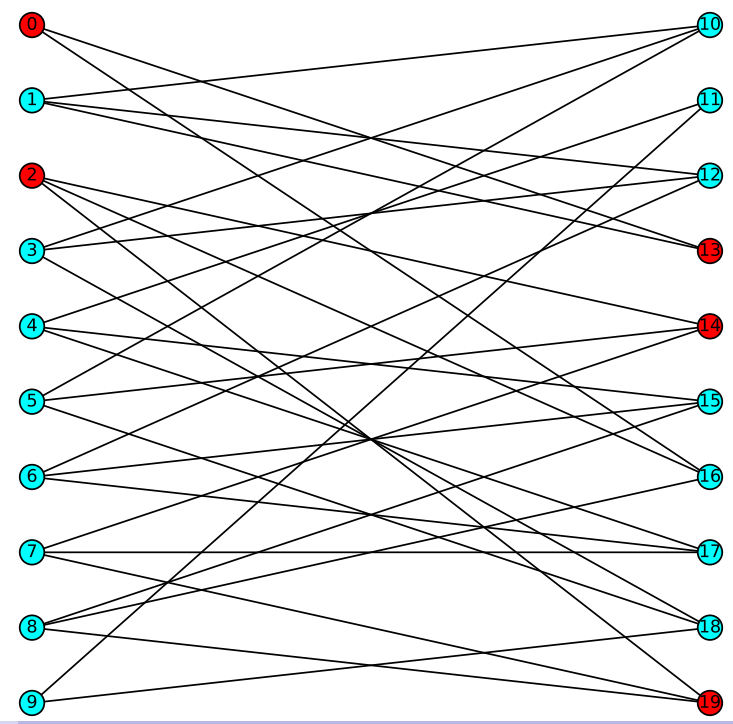

A. Grospellier

Efficient decoding of random errors for quantum expander codes 


\section{Decoding algorithm : first example}

Bits

Parity-check nodes

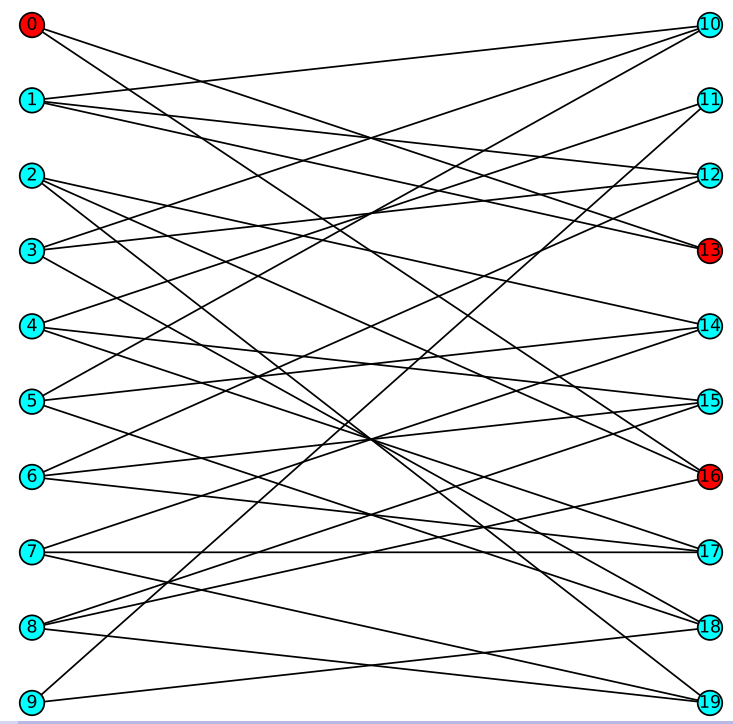

A. Grospellier

Efficient decoding of random errors for quantum expander codes 


\section{Decoding algorithm : first example}

Bits

Parity-check nodes

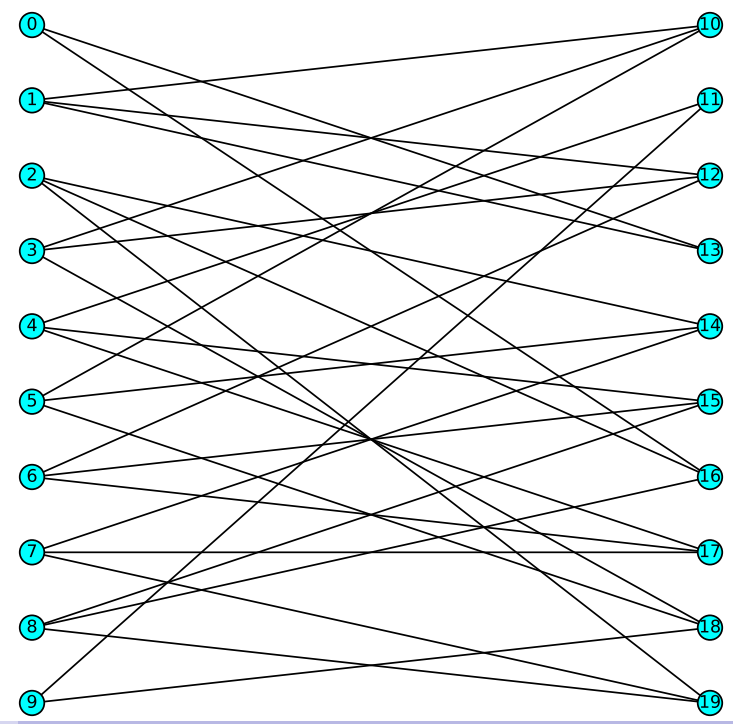

A. Grospellier

Efficient decoding of random errors for quantum expander codes 


\section{Decoding algorithm : second example}

Bits

Parity-check nodes

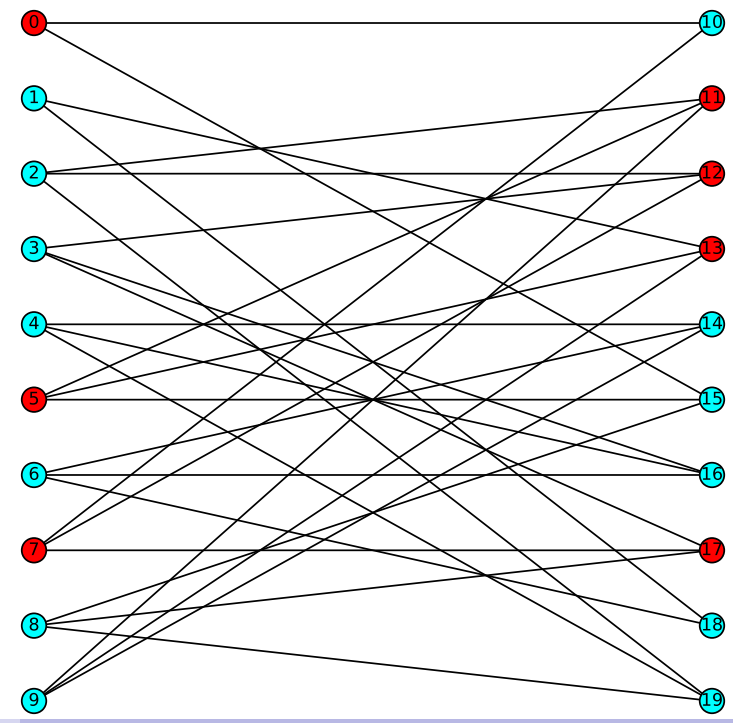

A. Grospellier

Efficient decoding of random errors for quantum expander codes

$10 / 23$ 


\section{Decoding algorithm : second example}

Bits

Parity-check nodes

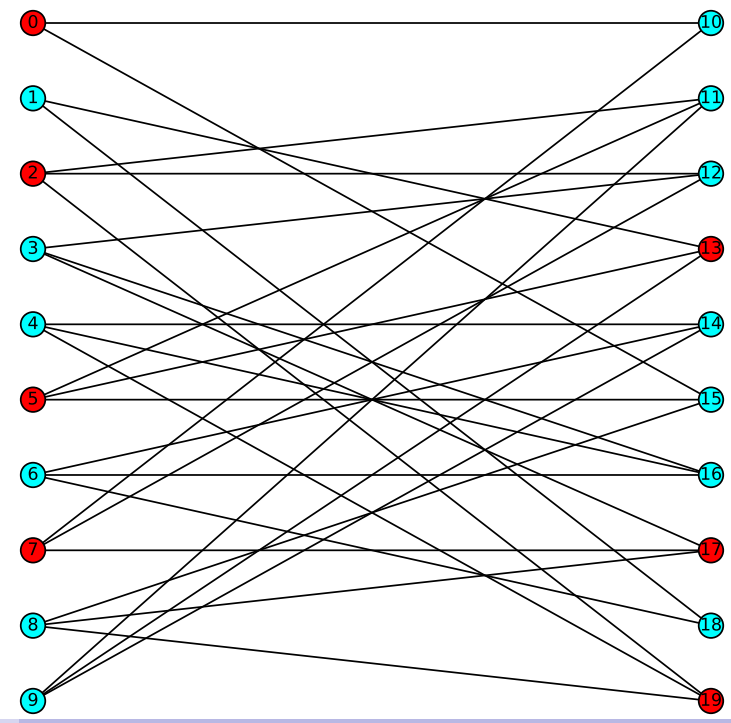

A. Grospellier

Efficient decoding of random errors for quantum expander codes

$10 / 23$ 


\section{Decoding algorithm : second example}

Bits

Parity-check nodes

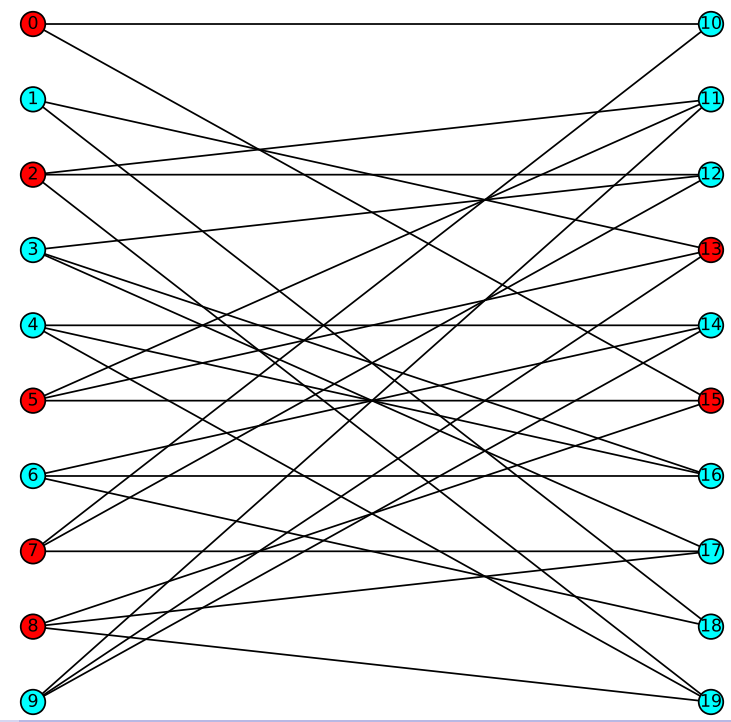

A. Grospellier

Efficient decoding of random errors for quantum expander codes

$10 / 23$ 


\section{Decoding algorithm : second example}

Bits

Parity-check nodes

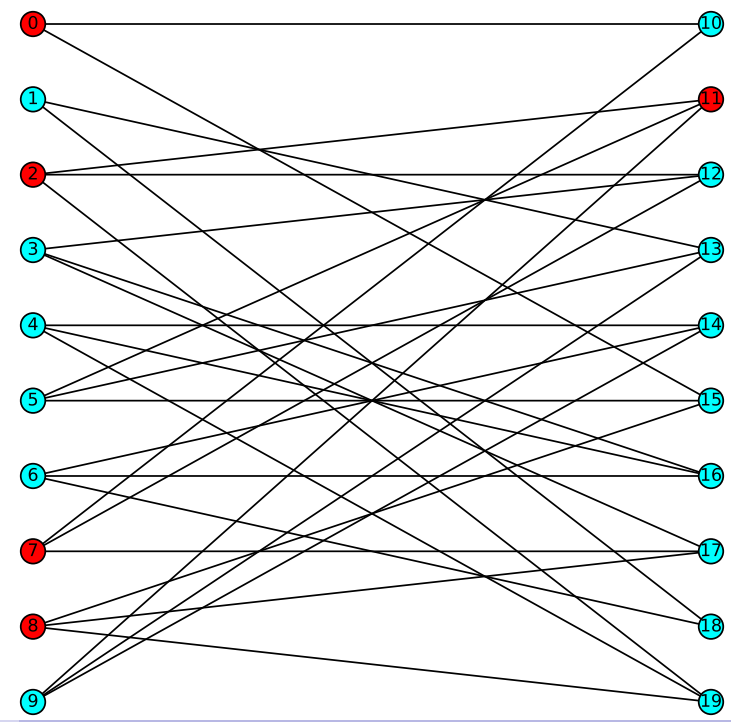

A. Grospellier

Efficient decoding of random errors for quantum expander codes

$10 / 23$ 


\section{Plan}

(1) Classical expander codes

(2) Quantum expander codes 


\section{From classical to quantum error correcting codes}

Hypergraph product [Tillich \& Zémor, '09]

Using a classical code $\mathcal{C}$, we can construct a $\llbracket n, k, d \rrbracket$-CSS code with :

- $k=\Theta(n)$

- $d=\Theta(\sqrt{n})$ : we can correct any error on $d$ Q-bits 


\section{From classical to quantum error correcting codes}

Hypergraph product [Tillich \& Zémor, '09]

Using a classical code $\mathcal{C}$, we can construct a $\llbracket n, k, d \rrbracket$-CSS code with :

- $k=\Theta(n)$

- $d=\Theta(\sqrt{n})$ : we can correct any error on $d$ Q-bits

\section{Theorem [Leverrier \& Tillich \& Zémor, '15]}

For the hypergraph product of an expander code $(\epsilon<1 / 6)$ :

There is an efficient decoding algorithm for this code.

This algorithm corrects any error of size $\leq \Theta(\sqrt{n})$

This algorithm is very close to the algorithm of Sipser and Spielman 


\section{Plan}

(1) Classical expander codes

(2) Quantum expander codes

(3) Our contribution 


\section{Our work}

- Question : What happens for random errors of size $\Theta(n)$ ?

- Depolarizing channel : each Q-bit has an X-type error (resp. Y,Z-type error) with probabilty $p$ independently 


\section{Our work}

- Question : What happens for random errors of size $\Theta(n)$ ?

- Depolarizing channel : each Q-bit has an X-type error (resp. Y,Z-type error) with probabilty $p$ independently

\section{Theorem : what we proved}

For a probability of error $p<10^{-16}$ :

$\lim _{n \rightarrow+\infty} \mathbb{P}(\mathcal{A}$ corrects the error $)=1$ 


\section{Our work}

- Question : What happens for random errors of size $\Theta(n)$ ?

- Depolarizing channel : each Q-bit has an X-type error (resp. Y,Z-type error) with probabilty $p$ independently

\section{Theorem : what we proved}

For a probability of error $p<10^{-16}$ :

$\lim _{n \rightarrow+\infty} \mathbb{P}(\mathcal{A}$ corrects the error $)=1$

Idea : The algorithm is local :

- If two errors are far they don't interact

- The initial error can be decomposed in clusters of size $O(\ln (n))$

- If there is no cluster of size $\Theta(\sqrt{n})$ during the algorithm, the error will be corrected 


\section{Locality of the algorithm : the adjacency graph}
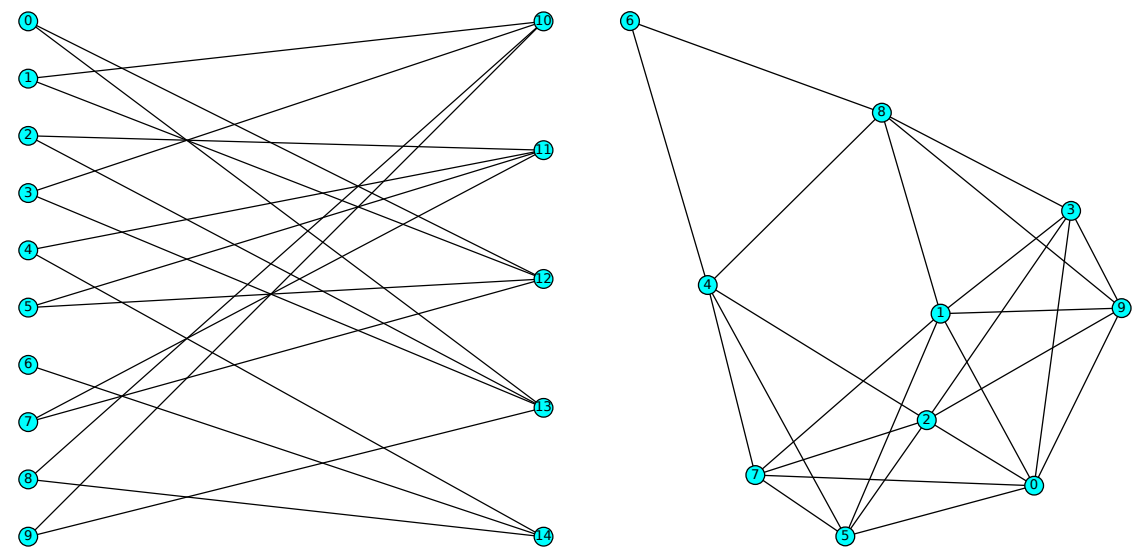


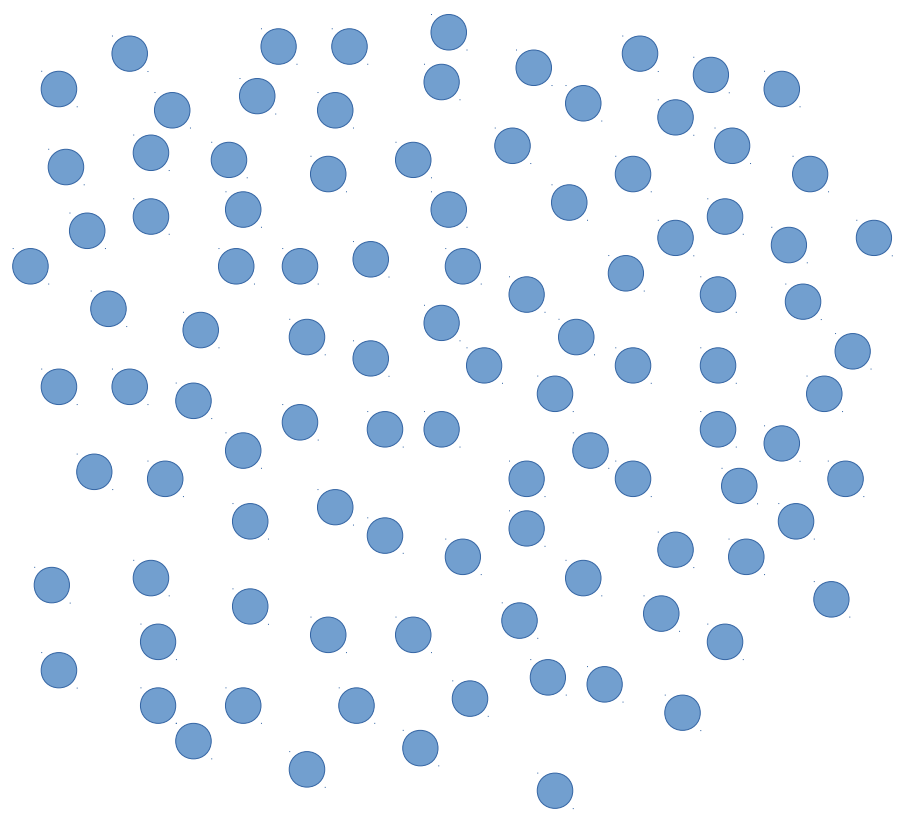




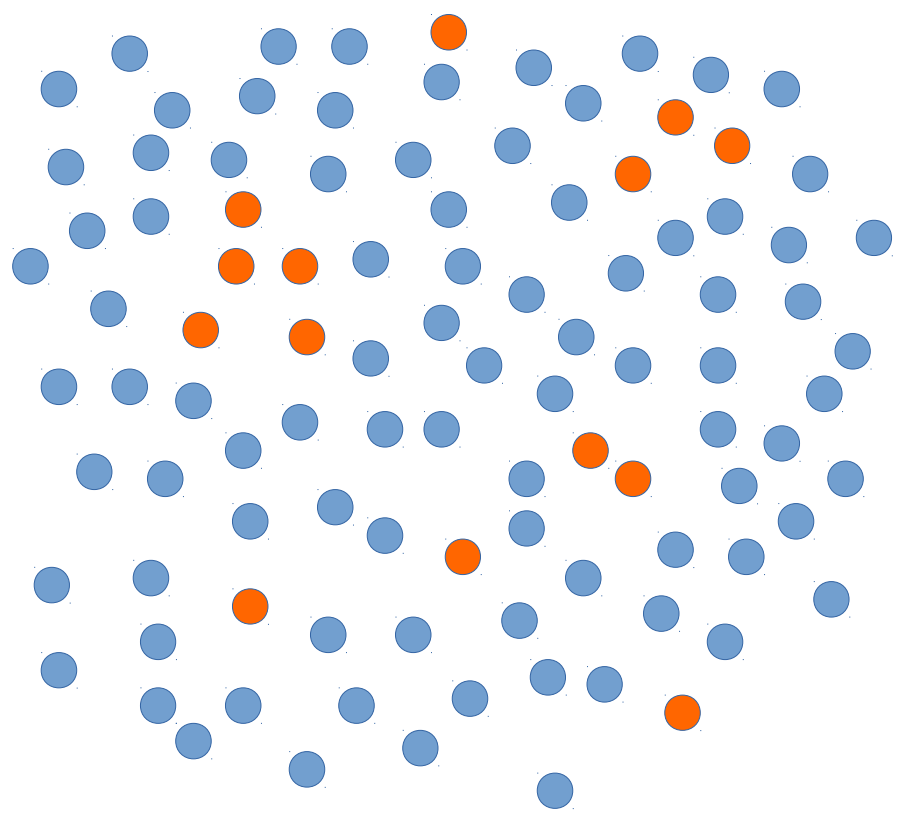




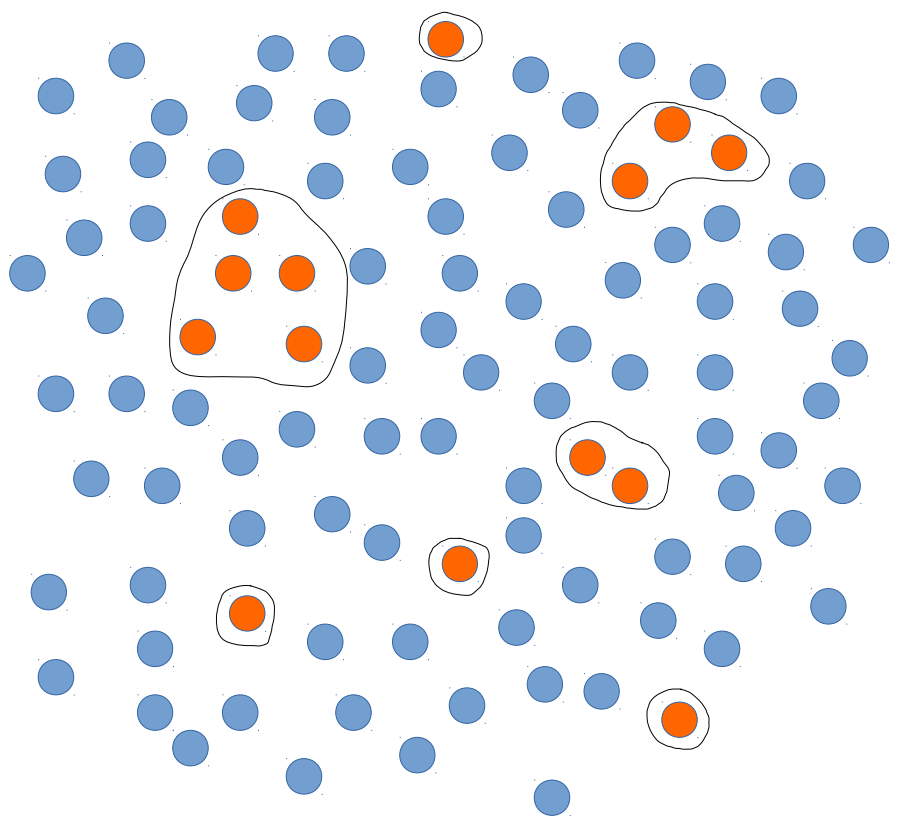




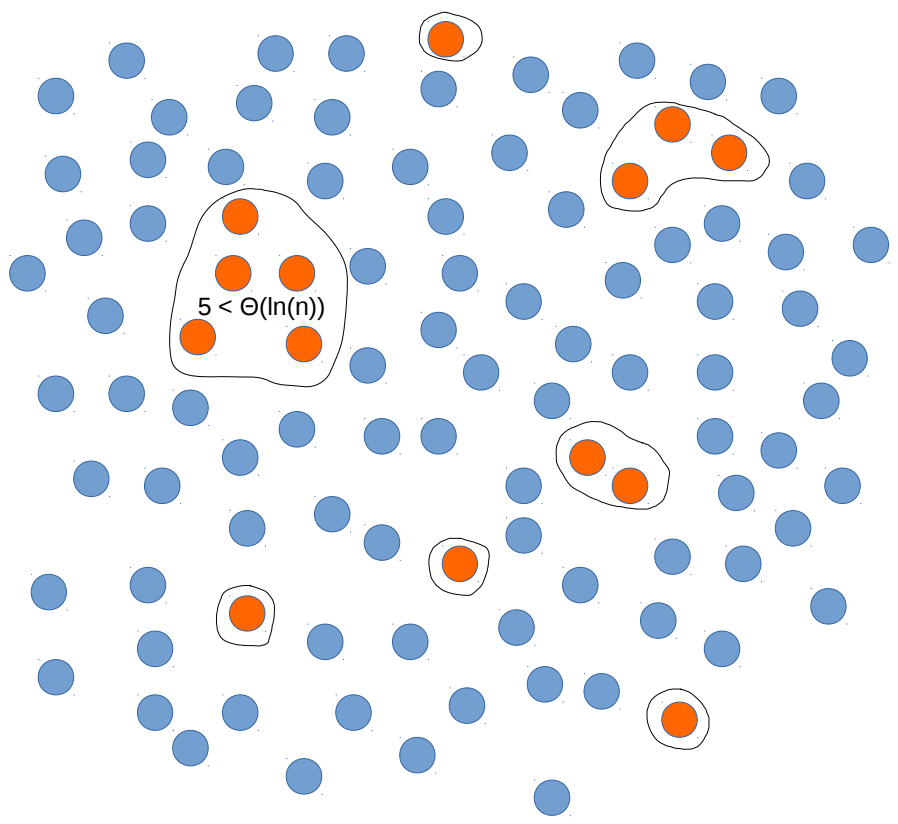




\section{In the following, we will say whp $P$ (with high probability the property $P$ holds) if : $\lim _{n \rightarrow+\infty} \mathbb{P}(P)=1$}


In the following, we will say whp $P$ (with high probability the property $P$ holds) if : $\lim _{n \rightarrow+\infty} \mathbb{P}(P)=1$

\section{Percolation Theorem}

For a probability of error $p<\frac{1}{d-1}$, whp :

- The size of any connected components is $\leq \Theta(\ln (n))$

For a probability of error $p>\frac{1}{d-1}$, whp :

- There is a connected component of size $\Theta(n)$ 
In the following, we will say whp $P$ (with high probability the property $P$ holds) if : $\lim _{n \rightarrow+\infty} \mathbb{P}(P)=1$

\section{Percolation Theorem}

For a probability of error $p<\frac{1}{d-1}$, whp :

- The size of any connected components is $\leq \Theta(\ln (n))$

For a probability of error $p>\frac{1}{d-1}$, whp :

- There is a connected component of size $\Theta(n)$

\section{Good news :}

The algorithm corrects any error of size $\leq \Theta(\sqrt{n})$

The algorithm corrects any error of size $\leq \Theta(\ln (n))$ 
In the following, we will say whp $P$ (with high probability the property $P$ holds) if : $\lim _{n \rightarrow+\infty} \mathbb{P}(P)=1$

\section{Percolation Theorem}

For a probability of error $p<\frac{1}{d-1}$, whp :

- The size of any connected components is $\leq \Theta(\ln (n))$

For a probability of error $p>\frac{1}{d-1}$, whp :

- There is a connected component of size $\Theta(n)$

\section{Good news :}

The algorithm corrects any error of size $\leq \Theta(\sqrt{n})$

The algorithm corrects any error of size $\leq \Theta(\ln (n))$

Problem : Some clusters can merge during the decoding 


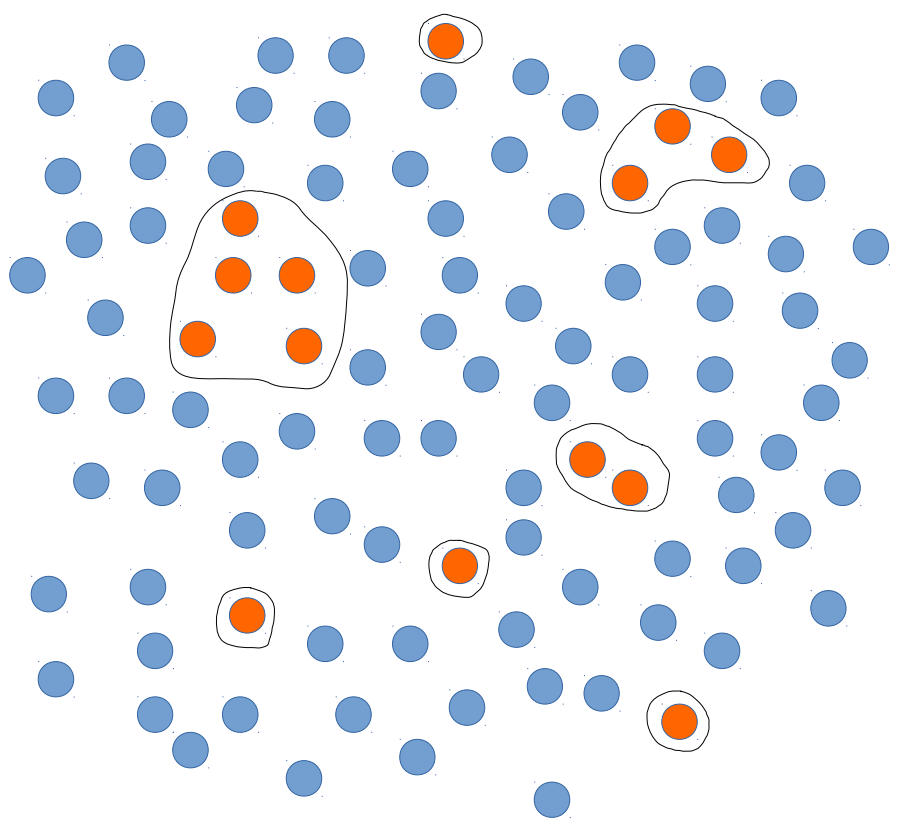




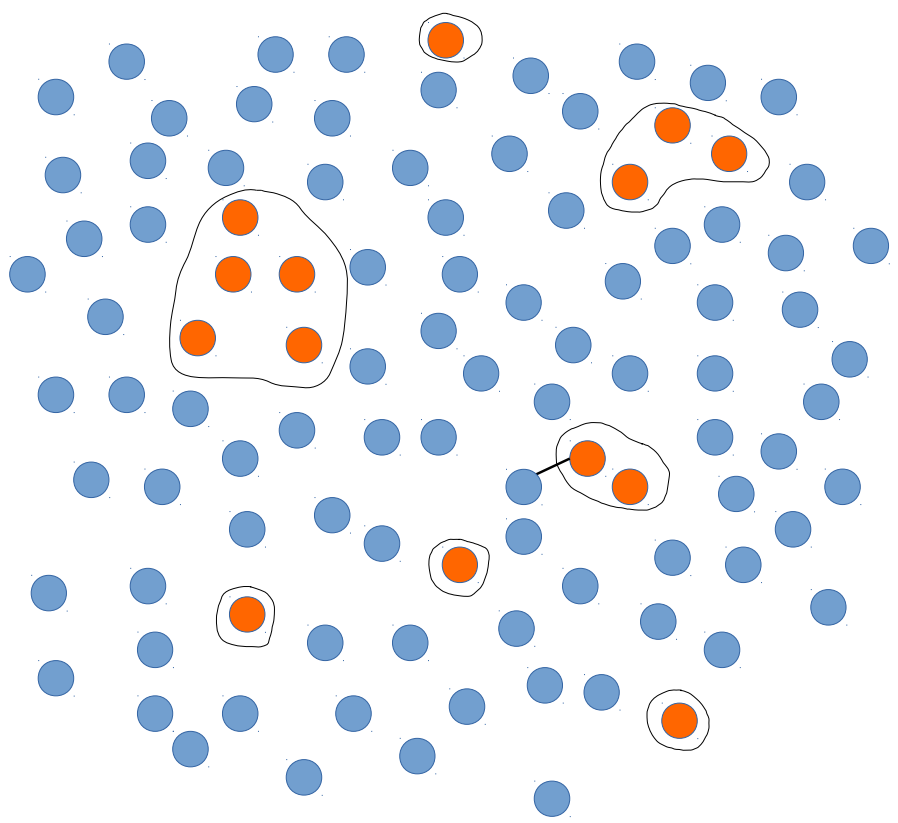




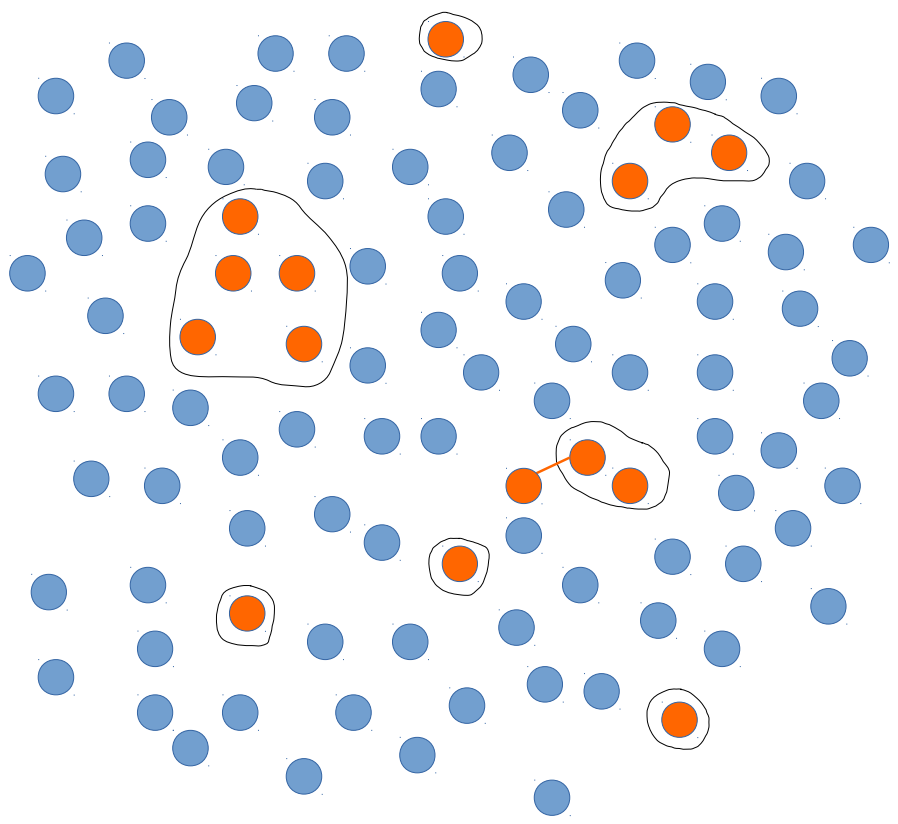




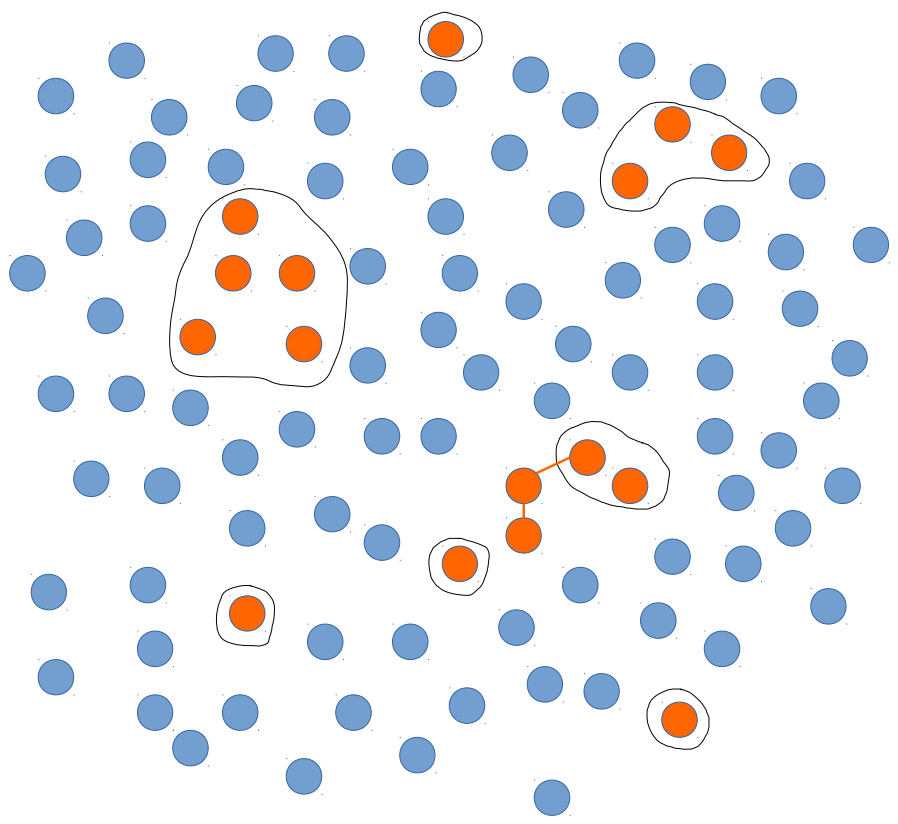




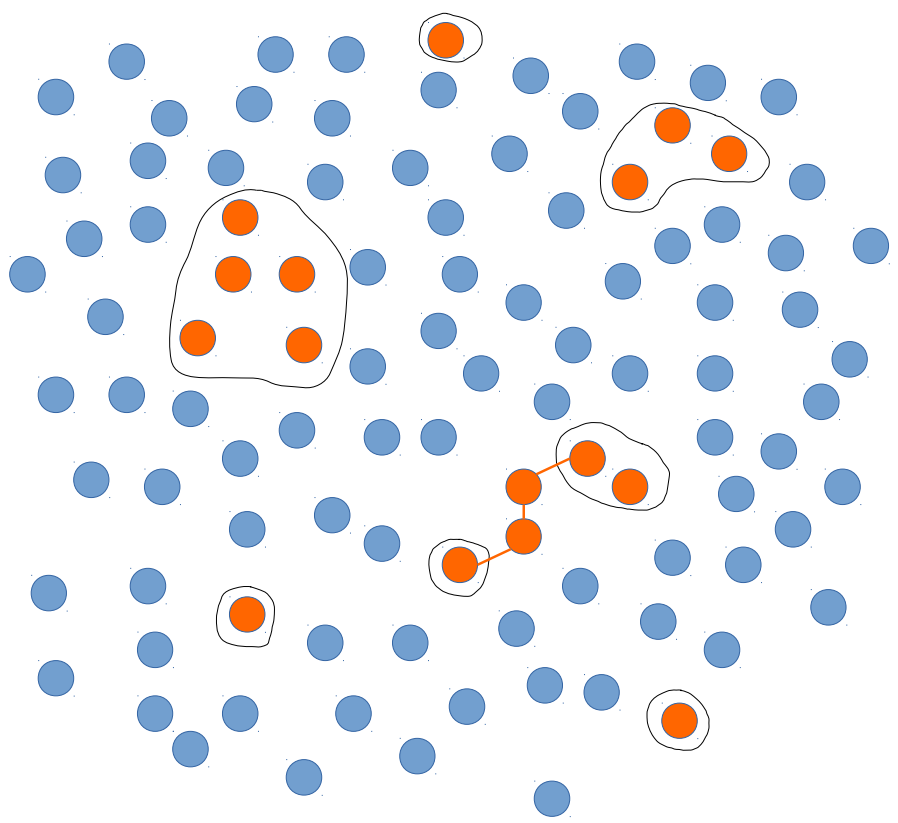




\section{Percolation Theorem}

For a probability of error $p<\frac{1}{d-1}$, whp :

- The size of any connected components is $\leq \Theta(\ln (n))$ 


\section{Percolation Theorem}

For a probability of error $p<\frac{1}{d-1}$, whp :

- The size of any connected components is $\leq \Theta(\ln (n))$

\section{Percolation theorem, reformulation}

For a probability of error $p<\frac{1}{d-1}$, whp :

- if $|X \cap E(p)| \geq 1 \times|X|$ then $|X|<\Theta(\ln (n))$ 


\section{Percolation Theorem}

For a probability of error $p<\frac{1}{d-1}$, whp :

- The size of any connected components is $\leq \Theta(\ln (n))$

\section{Percolation theorem, reformulation}

For a probability of error $p<\frac{1}{d-1}$, whp :

- if $|X \cap E(p)| \geq 1 \times|X|$ then $|X|<\Theta(\ln (n))$

\section{Percolation theorem, generalisation}

$$
\begin{aligned}
& \forall \alpha>0, \text { if } p<\operatorname{cst}(\alpha, d) \text {, whp : } \\
& \text { - if }|X \cap E(p)| \geq \alpha \times|X| \text { then }|X|<\Theta(\ln (n))
\end{aligned}
$$




\section{Percolation Theorem}

For a probability of error $p<\frac{1}{d-1}$, whp :

- The size of any connected components is $\leq \Theta(\ln (n))$

\section{Percolation theorem, reformulation}

For a probability of error $p<\frac{1}{d-1}$, whp :

- if $|X \cap E(p)| \geq 1 \times|X|$ then $|X|<\Theta(\ln (n))$

Percolation theorem, generalisation

$\forall \alpha>0$, if $p<\operatorname{cst}(\alpha, d)$, whp :

- if $|X \cap E(p)| \geq \alpha \times|X|$ then $|X|<\Theta(\ln (n))$

Complexity of the decoding algorithm

The number of flips is linear in the size of the initial error 


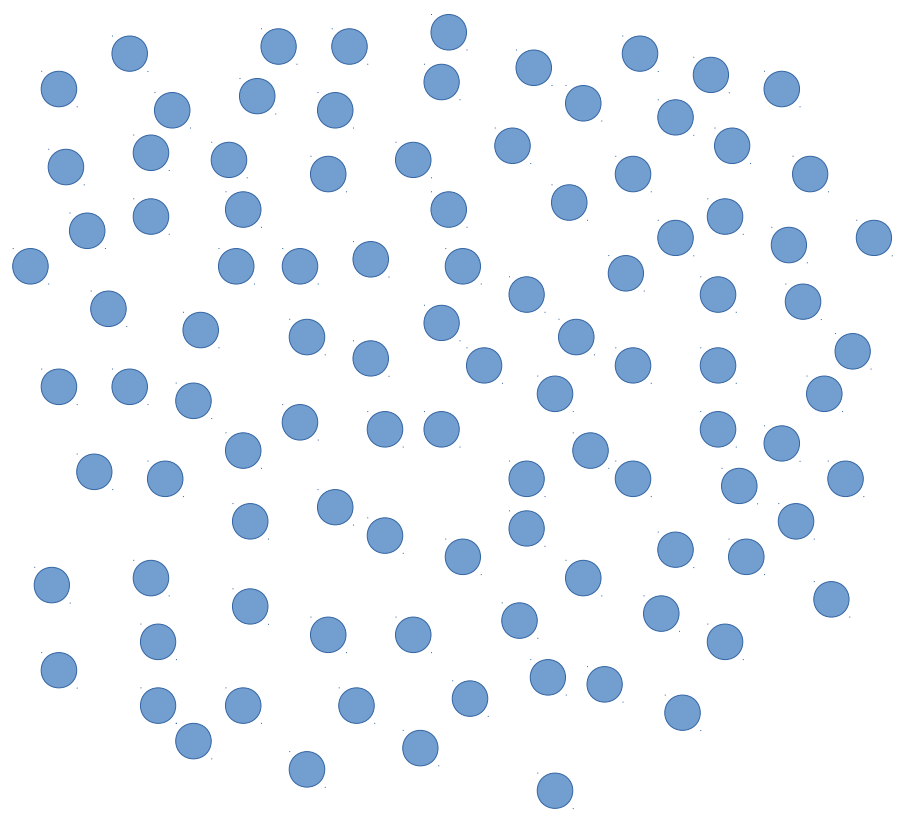




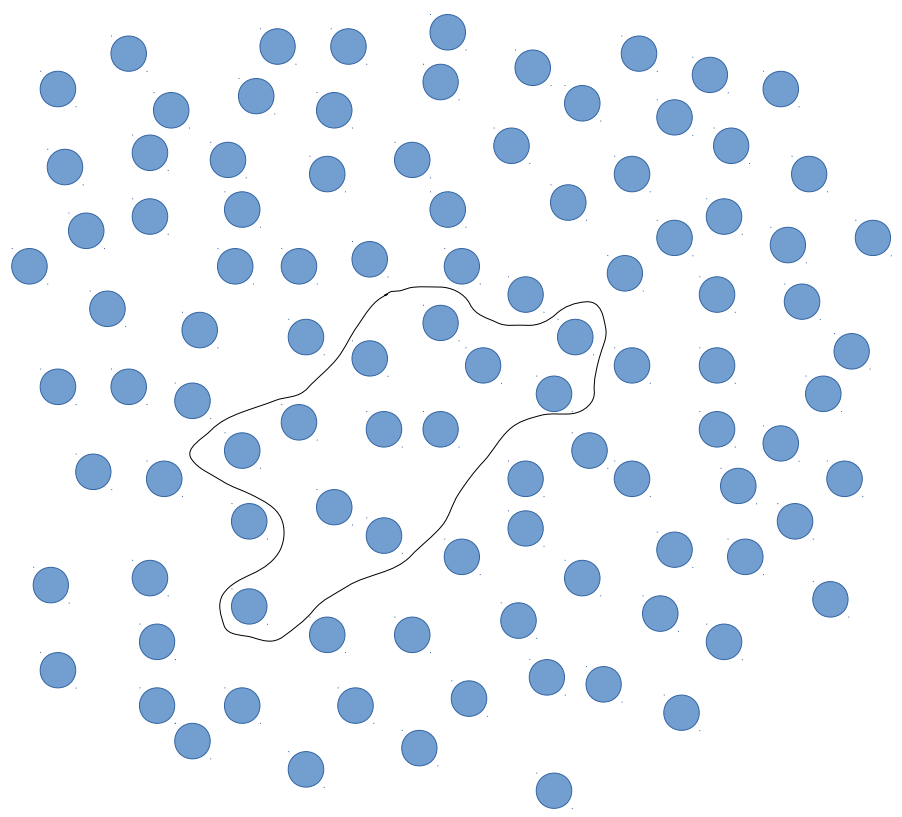




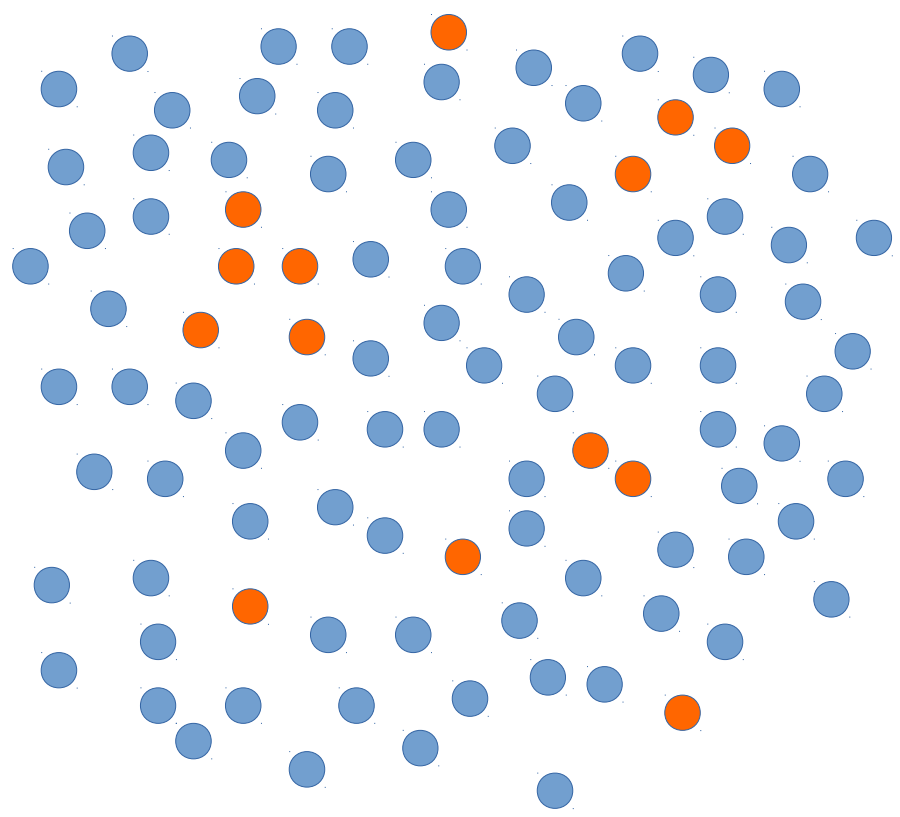




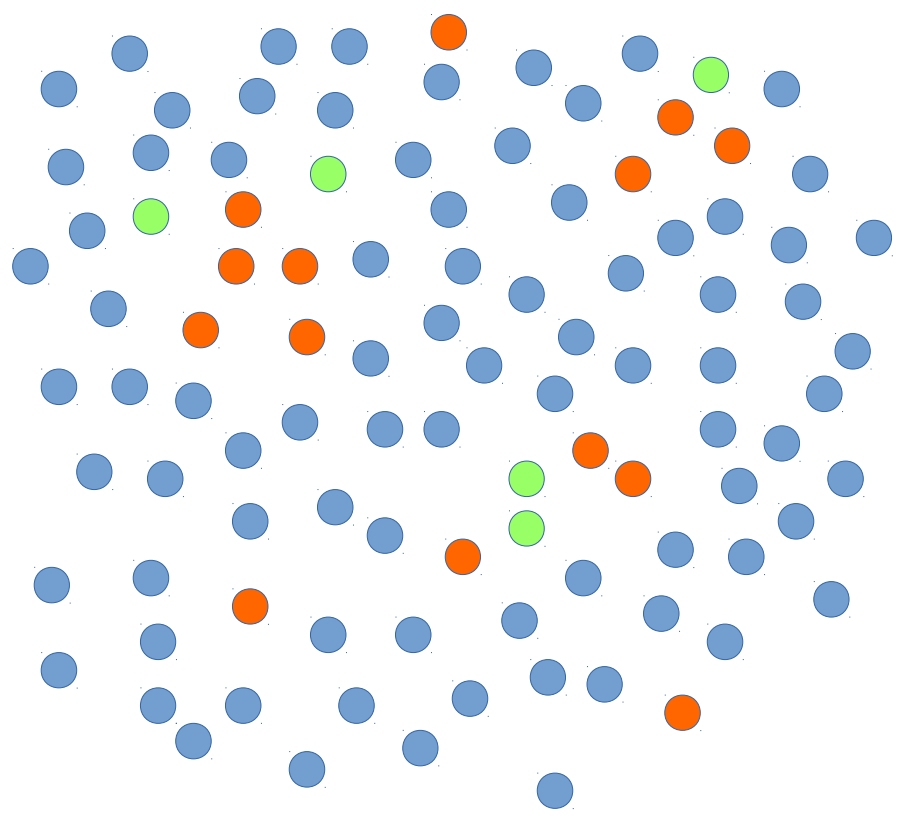




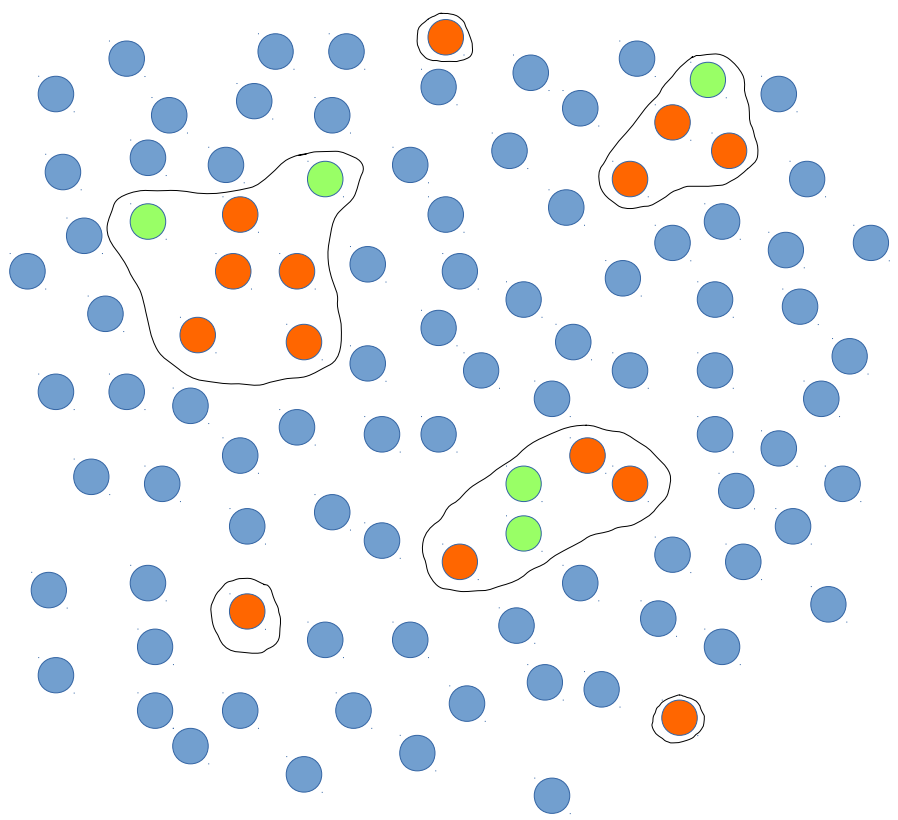




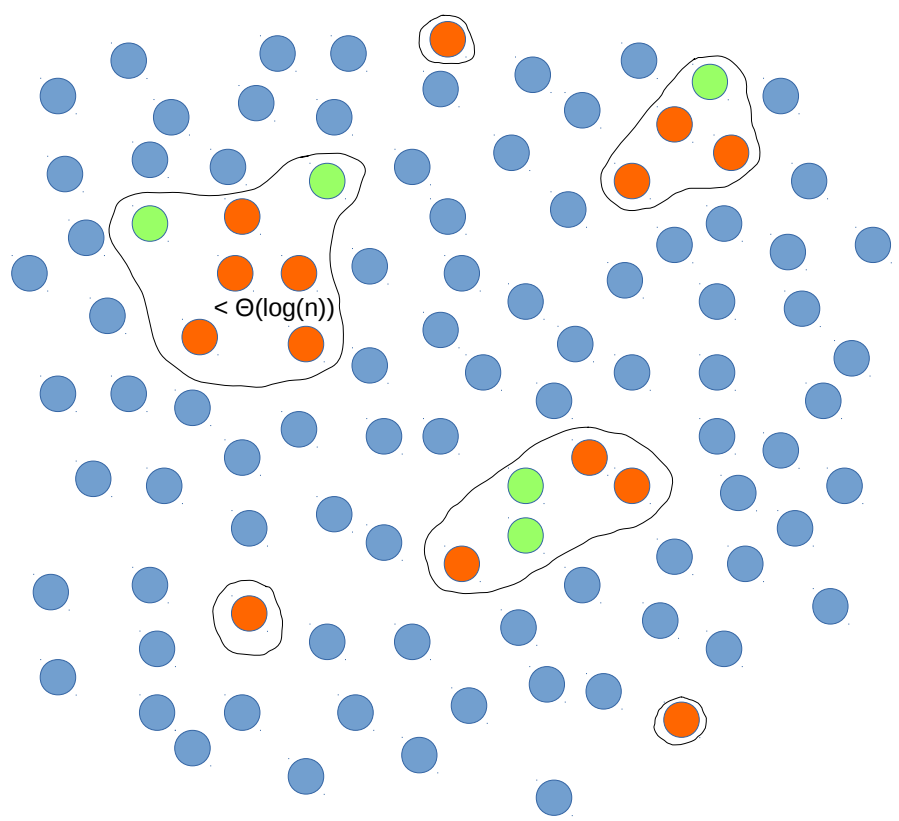




\section{Theorem : what we proved}

For a probability of error $p<10^{-16}$ : $\lim _{n \rightarrow+\infty} \mathbb{P}(\mathcal{A}$ corrects the error $)=1$ 


\section{Theorem : what we proved}

For a probability of error $p<10^{-16}$ :

$\lim _{n \rightarrow+\infty} \mathbb{P}(\mathcal{A}$ corrects the error $)=1$

\section{Ideas to improve this bound :}

(2) Improve the bound in the percolation theorem :

What is the critical probability?

(2) Restrict the proof to interesting clusters:

* The diameter of an interesting cluster is $\leq \Theta(\ln (\ln (n)))$

* The number of edges inside an interesting cluster is large (ideas from bootstrap percolation) 


\section{Conclusion}

The hypergraph product of an expander code :

- is an LDPC quantum code

- has a constant rate

- has a minimal distance : $d=\Theta(\sqrt{n})$

The decoding algorithm :

- has a capacity of correction : $\Theta(\sqrt{n})$

- corrects the error with high probability for the depolarizing channel

Futur work :

- improve our bound

- run simulations

- apply this result to fault tolerant quantum computation (Gottesman) 


\section{Thank you for your attention}




\section{A motivation : fault-tolerant quantum computation}

Threshold Theorem [Ben-Or \& Aharonov, '97]

We can simulate a quantum circuit with perfect gates by a circuit with noisy gates of size quasi-linear

Theorem : what we proved

For a probability of error $p<10^{-16}$ :

$\lim _{n \rightarrow+\infty} \mathbb{P}(\mathcal{A}$ corrects the error $)=1$

$n \rightarrow+\infty$

What we hope to prove using [Gottesman, '13]

We can simulate a quantum circuit with perfect gates by a circuit with noisy gates of size linear 


\section{$n=10, m=5, d_{1}=2, d_{2}=\frac{n \times d_{1}}{m}=4$}

(2)

(1)

(2)

(3)

(4)

(5)

(6)

(7)

(8)

(9) 
$n=10, m=5, d_{1}=2, d_{2}=\frac{n \times d_{1}}{m}=4$

()

(10)

(1)

(2)

(11)

(3)

(4)

(12)

(5)

(6)

(7)

(13)

(8)

(9)

(14) 
$n=10, m=5, d_{1}=2, d_{2}=\frac{n \times d_{1}}{m}=4$

(ㄱ)

(1)

(1) 트

(2) 트

(11)

(3) 트

(4) 트

(12)

(5) 르

(ㄷ) 트

(ㄱ) 트

(13)

(8) 틈

(9)

(14) 
$n=10, m=5, d_{1}=2, d_{2}=\frac{n \times d_{1}}{m}=4$

(ㅇ)

코

(1)

(2)

$\square(11)$

(3)

(4)

(5)

च 12

(6)

(7)

三。

$0=$

(9)

$\vdots$ 
$n=10, m=5, d_{1}=2, d_{2}=\frac{n \times d_{1}}{m}=4$

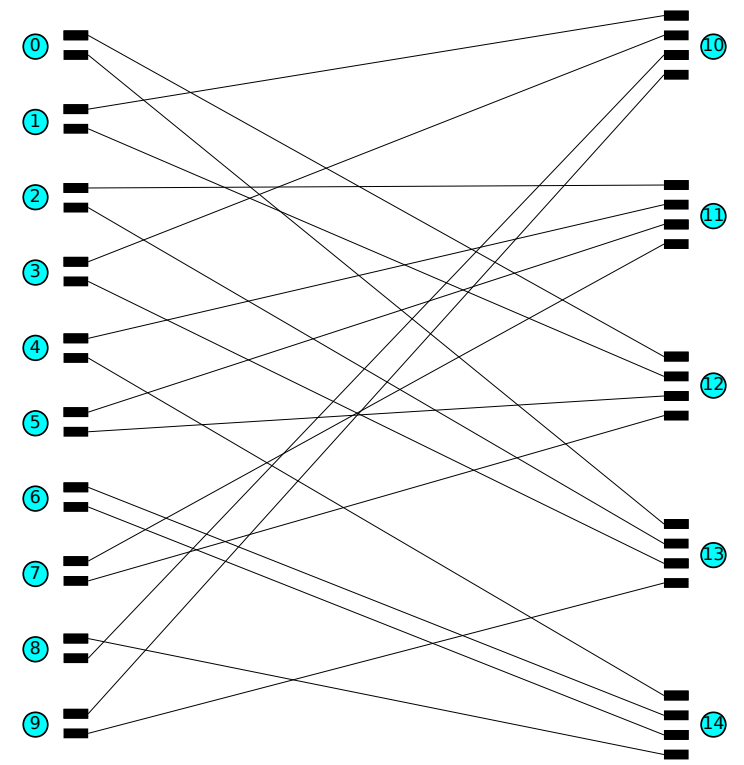


$n=10, m=5, d_{1}=2, d_{2}=\frac{n \times d_{1}}{m}=4$

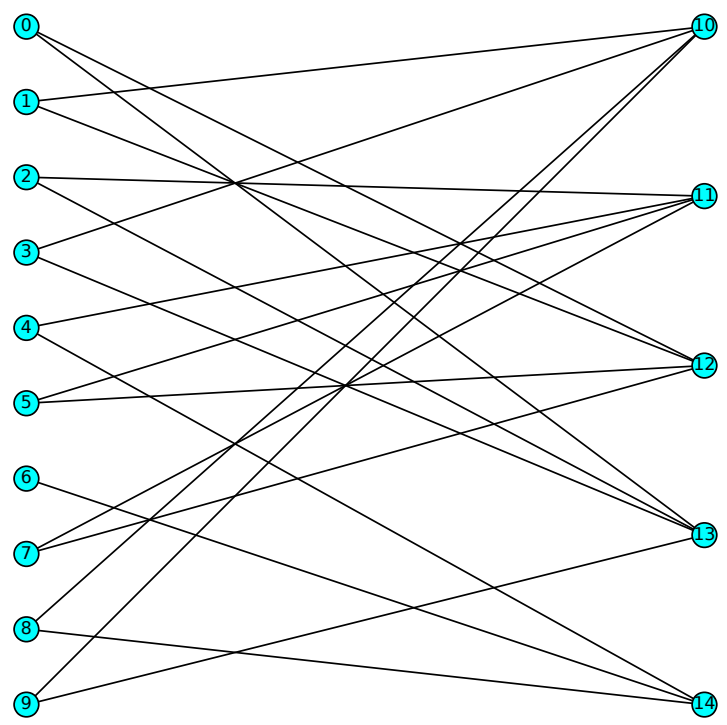

